\title{
The efficacy and safety of S-1-based regimens in the first-line treatment of advanced gastric cancer: a systematic review and meta-analysis
}

\author{
Emil ter Veer ${ }^{1} \cdot$ Nadia Haj Mohammad ${ }^{1} \cdot$ Paul Lodder $^{2} \cdot$ Lok Lam Ngai $^{1}$. \\ Mary Samaan ${ }^{1}$ Martijn G. H. van Oijen ${ }^{1}$ Hanneke W. M. van Laarhoven ${ }^{1}$
}

Received: 4 September 2015/Accepted: 7 December 2015/Published online: 11 January 2016

(C) The Author(s) 2016. This article is published with open access at Springerlink.com

\begin{abstract}
Background S-1 is first-line therapy for advanced gastric cancer in Asia and is used with increased frequency in Western counties. We conducted a meta-analysis to investigate the efficacy and toxicity of S-1-based therapy compared with 5-fluorouracil (5-FU)/capecitabine-based therapy and S-1-based combination therapy compared with S-1 monotherapy.

Methods MEDLINE, Embase, the Cochrane Central Register of Controlled Trials, American Society of Clinical Oncology meeting abstracts, European Society for Medical Oncology meeting abstracts and ClinicalTrials.gov were searched for randomized clinical trials until May 2015. Data were extracted for overall survival (OS), progressionfree-survival (PFS), objective response rate (ORR) and grade 1-2 and grade 3-4 adverse events. Stratified OS data for subgroups were extracted.
\end{abstract}

PROSPERO registration: The protocol of this systematic review was published in the international database of prospectively registered systematic reviews (PROSPERO) with registration number CRD42014010654 (http://www.crd.york.ac.uk/PROSPERO/display_ record.asp?ID=CRD42014010654).

Electronic supplementary material The online version of this article (doi:10.1007/s10120-015-0587-8) contains supplementary material, which is available to authorized users.

Hanneke W. M. van Laarhoven

h.vanlaarhoven@amc.uva.nl

1 Department of Medical Oncology, Academic Medical Centre, University of Amsterdam, Meibergdreef 9, F4-224,

1105 AZ Amsterdam, The Netherlands

2 Department of Psychology, University of Amsterdam, Amsterdam, The Netherlands
Results S-1 was not different from 5-FU (eight studies, $n=2788$ ) in terms of OS [hazard ratio (HR) $0.93,95 \%$ confidence interval (CI) 0.85-1.01] and PFS (HR 0.87, $95 \%$ CI 0.73-1.04), whereas ORR was higher (risk ratio $1.43,95 \%$ CI 1.05-1.96). There was no subgroup difference in efficacy among Asian and Western patients, but in Western patients S-1 was associated with a lower rate of febrile neutropenia, toxicity-related deaths and grade 3-4 stomatitis and mucositis compared with 5-FU. S-1 showed no difference in efficacy compared with capecitabine (three studies, $n=329$ ), but was associated with a lower rate of grade 3-4 neutropenia and grade 1-2 hand-foot syndrome. S-1-combination therapy was superior to S-1 monotherapy (eight studies, $n=1808$ ) in terms of OS (HR 0.76, $95 \%$ CI 0.65-0.90), PFS (HR 0.68, $95 \%$ CI 0.56-0.82) and ORR (risk ratio $1.20,95 \%$ CI 1.04-1.38) but was more toxic. Survival benefit of S-1 combination therapy over S-1 monotherapy was most pronounced in patients with nonmeasurable disease, diffuse-type histological features and peritoneal metastasis.

Conclusions $\mathrm{S}-1$ is effective and tolerable as first-line therapy for advanced gastric cancer in both Asian and Western countries.

Keywords Advanced gastric cancer $\cdot$ S-1 .

Chemotherapy $\cdot$ Meta-analysis

\section{Introduction}

Fluoropyrimidines are the backbone of first-line therapy for advanced gastric cancer [1,2]. The novel fluoropyrimidine S-1 has quickly become the standard of care in Asia, but there is uncertainty about the role of S-1 in Western countries. Although S-1 is used with increasing frequency 
in Western countries, it has not fully replaced 5-fluorouracil (5-FU) and capecitabine. Meta-analyses have shown a marginally significant prolonged survival time and higher response rates for S-1 therapy compared with 5-FU therapy [3-5] but not for S-1 therapy compared with capecitabine therapy [6-9]. However, some of these reviews included retrospective studies, which may lead to bias of the overall effect observed or did not incorporate the newest evidence in this field [10-15]. For example, in addition to the FLAGS trial [16], which was conducted in Western countries, the recently presented DIGEST trial [11] can also shed light on the role of S-1 therapy in Western patients.

The use of doublets of cytotoxic agents versus singlets is associated with prolonged survival [17] and therefore S-1based combination therapy versus S-1 monotherapy has been investigated in several large trials in Asia. Previous meta-analyses have indicated that combination therapy significantly prolonged survival over monotherapy, but generally combination therapy was more toxic $[18,19]$. However, the final results of four randomized studies, including the pivotal START trial, which was the first phase III trial to compare S-1 combined with a taxane with S-1 alone, were not included in these reviews [12, 13, 20, 21]. Moreover, it is also still an open question if there are predictive factors to define which patient subgroups will benefit most from S-1 combination therapy compared with S-1 monotherapy.

Therefore, the objectives of our study were to systematically review all available literature on randomized clinical trials to investigate the efficacy and toxicity by means of meta-analysis of S-1-based therapy compared with 5-FUand capecitabine-based therapy and of S-1-based combination therapy compared with S-1 monotherapy.

\section{Methods}

\section{Study protocol}

The protocol of this review has been published in the international prospective register of systematic reviews (PROSPERO): http://www.crd.york.ac.uk/PROSPERO/dis play_record.asp?ID=CRD42014010654.

\section{Literature search}

For the searching of the electronic databases [MEDLINE, Embase and Cochrane Central Register of Controlled Trials (CENTRAL)], a sensitive search strategy without date restriction was applied using the medical subject headings of 'S-1' and 'gastric cancer'; thereafter, the results were filtered for clinical trials. ClinicalTrials.gov (http://www. clinicaltrials.gov) was searched for the term ' $\mathrm{S}-1$ ' within the topic 'stomach neoplasm' and the results were filtered for phase II and phase III trials. In addition, all meeting abstracts from the American Society of Clinical Oncology and European Society for Medical Oncology up to May 2015 were searched via http://www.ascopubs.org/search and http://annonc.oxfordjournals.org/search, respectively, for the following terms: 'S-1' and 'gastric'. The full search history is available in Document S1 in the electronic supplementary material. Two reviewers (E.t.V. and M.S.) reviewed the literature independently, and discrepancies were resolved by discussion with an arbiter (N.H.M.) until consensus was reached. This systematic review was performed according to the Preferred Reporting Items for Systematic Reviews and Meta-analyses (PRISMA) statement.

\section{Inclusion criteria}

Studies had to meet the following eligibility criteria: (1) included patients with pathologically proven advanced gastric cancer (recurrent or unresectable disease); (2) firstline palliative (a) S-1-based therapy (monotherapy or doublet therapy) compared with 5-FU- or capecitabinebased chemotherapy (monotherapy or doublet therapy) or (b) S-1-based combination chemotherapy compared with S-1 monotherapy; and (3) prospective phase II or phase III randomized controlled trials.

\section{Outcomes and data extraction}

The primary efficacy outcome was overall survival (OS). To identify potential predictive factors for the efficacy of S-1 combination therapy compared with S-1 monotherapy, subgroup data were extracted for OS if possible. Secondary efficacy outcomes were progression-free survival (PFS) and overall response rate (ORR), defined as the sum of both partial and complete responses according to the Response Evaluation Criteria in Solid Tumors (RECIST). Tolerability outcomes comprised the incidence of adverse events (AEs) divided into mild toxicity (grade 1-2 AEs) and severe toxicity (grade 3-4 AEs). In all studies, AEs were scored according to the National Cancer Institute Common Toxicity Criteria (http://ctep.cancer.gov). Two reviewers (E.t.V. and N.H.M.) were involved in data extraction; discrepancies were resolved by discussion with an arbiter (L.N.) until consensus was reached.

\section{Study quality assessment}

Two reviewers (E.t.V. and N.H.M.) independently examined the quality of all included studies using the Cochrane risk of bias tool (Cochrane Handbook for Systematic 


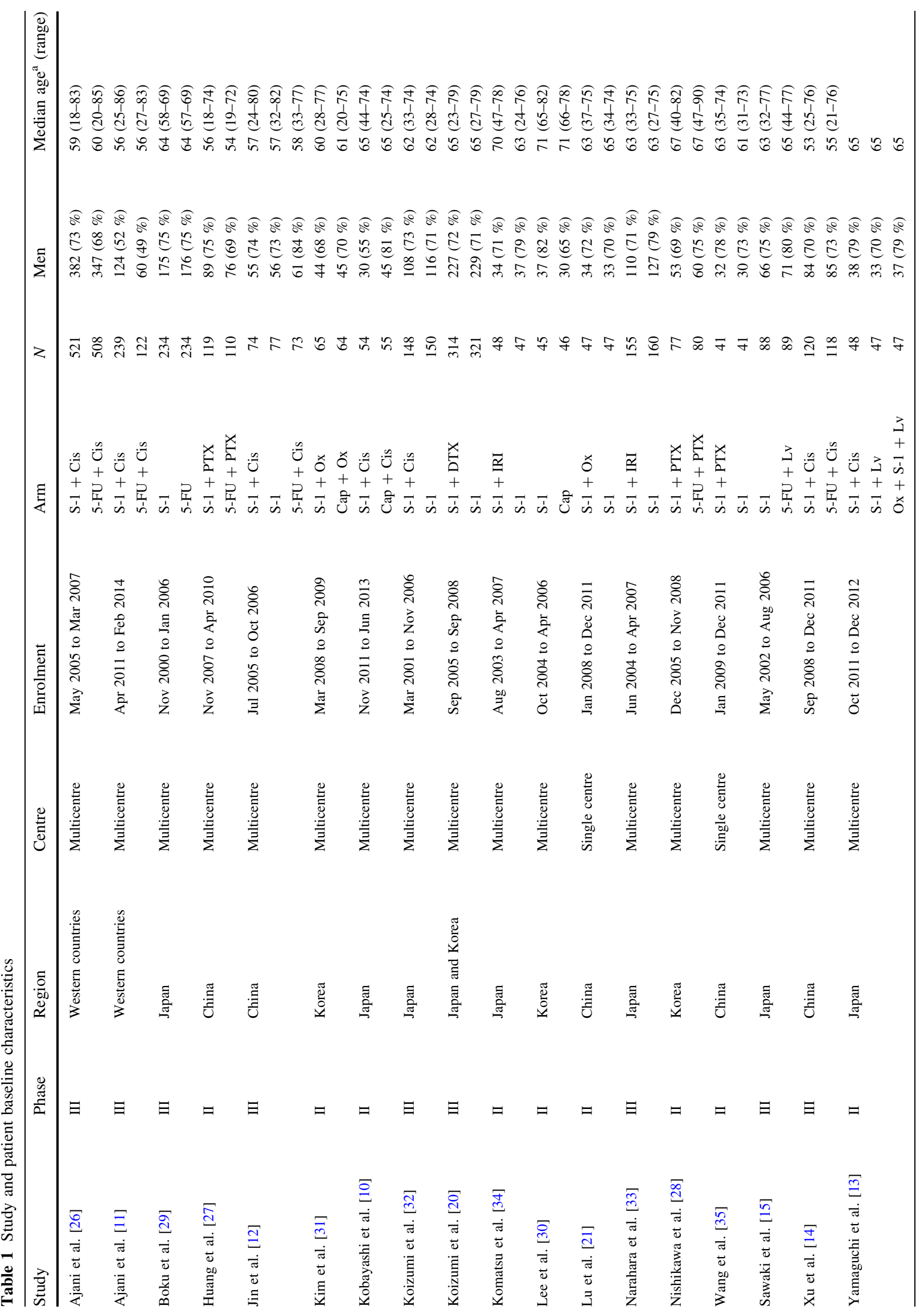




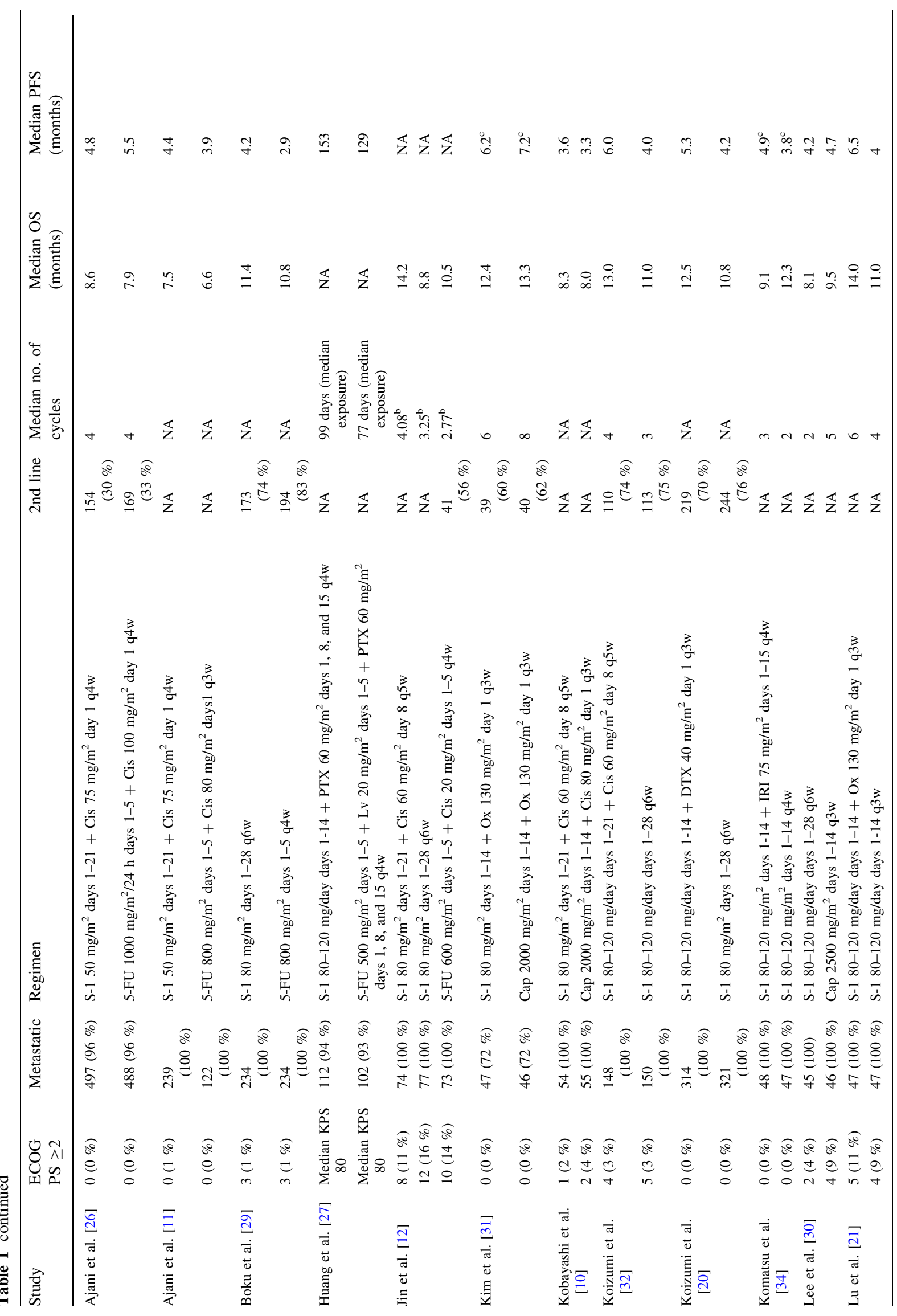




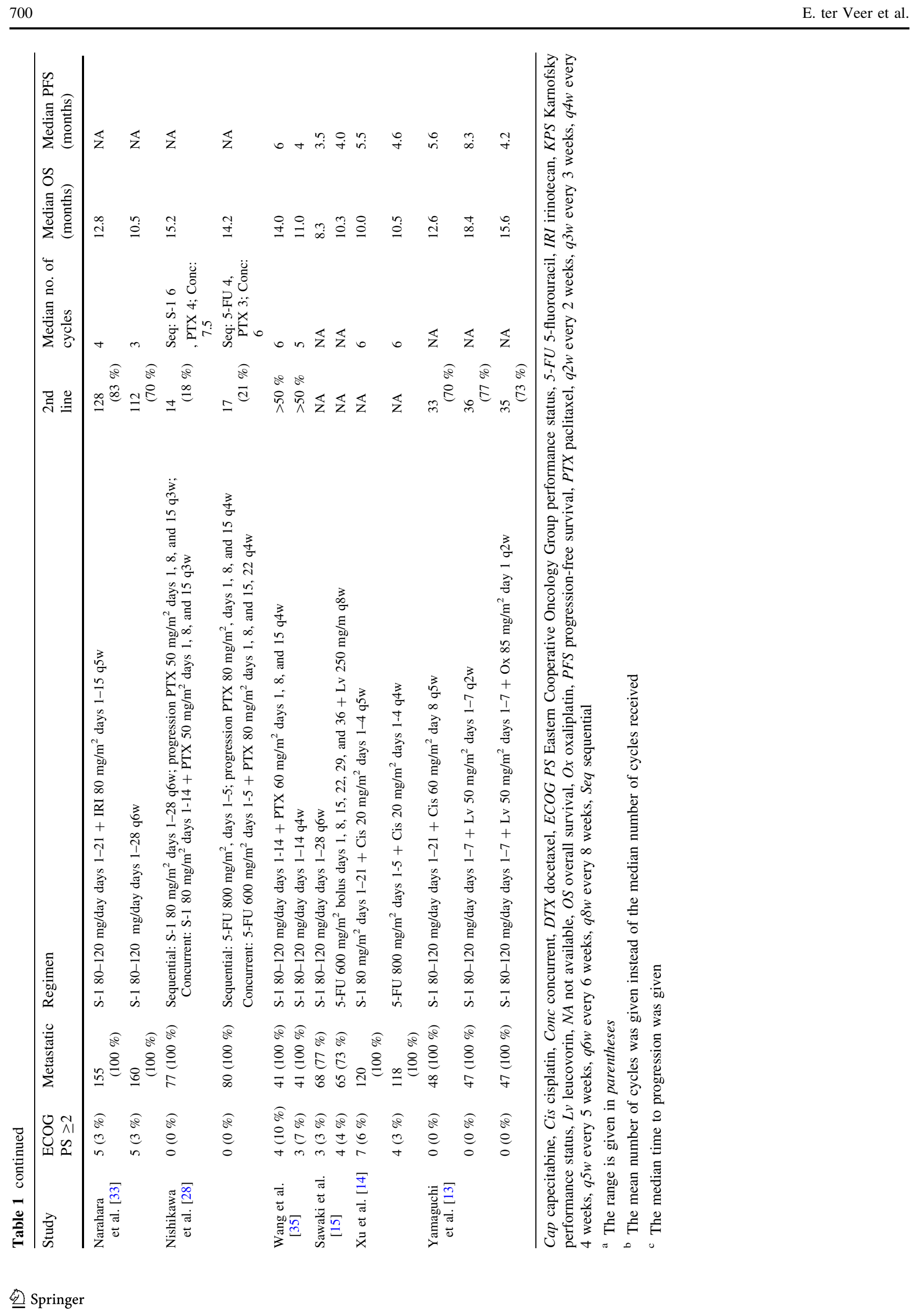


Reviews of Interventions, version 5.1.0) until consensus was reached. Studies with a high risk of bias were not included in the analysis. Since the primary outcome, OS, would not be influenced by the absence of a blinded imaging review, this item was not scored as unknown or high risk of bias for OS. Single-centre studies and studies without a published full article were rated as unclear risk of other possible bias. To assess the effect of study quality on the pooled estimate, sensitivity analyses were conducted by (1) omission of studies described in conference reports only and (2) omission of studies stepwise according to unknown risk of bias rating on one item, on two items and on three or more items.

\section{Statistical analysis}

Pairwise meta-analyses using random-effect models were conducted with the Metagen $\mathrm{R}$ package [22] and Review Manager 5.3. For OS and PFS outcomes, hazard ratios (HRs) and $95 \%$ confidence intervals (CIs) were extracted by the method described by Tierney et al. [23]. An HR less than 1 indicates a beneficial effect for the experimental arm, and an HR of 0.80 or less was considered clinically meaningful [24]. In addition, stratified HRs for OS in the patient subgroups were pooled with meta-analysis, and subgroup differences were statistically tested with chisquare tests. Risk ratios (RRs) were calculated for ORR (an RR greater than 1 indicates a beneficial effect for the experimental arm) and for event counts of grade 1-2 and grade 3-4 toxicity in both arms (an RR less than 1 indicates a beneficial effect for the experimental arm).

Statistical heterogeneity was tested with the Cochran Q test and quantified by the $I^{2}$ index. Substantial heterogeneity $\left(I^{2} \geq 30 \%\right)$ was explored by subgroup and sensitivity analyses. We tested for funnel plot asymmetry by regressing study outcomes on the standard error of the effect size [25]. All analyses were based on the intentionto-treat population of the included studies. All tests were performed two-sided, and a $P$ value of less 0.05 was considered statistically significant.

\section{Results}

\section{Literature search and study quality}

Three hundred and fifty-four unique references were identified through our searching MEDLINE, Embase and CENTRAL until May 2015, from which 326 were excluded after abstract screening, because of ineligibility according to the criteria for this review. Of the 28 reports remaining for full-text screening, four studies were eligible to assess S-1-based versus 5-FU-based therapy [26-29],
Fig. 1 S-1-based therapy compared with 5-fluorouracil (5-FU)- and capecitabine ( $\mathrm{Cap}$ )-based therapy: a overall survival; b progressionfree survival; c overall response rate. $C I$ confidence interval, $C$ is cisplatin, $d f$ degrees of freedom, $E$ effect, $H$ heterogeneity, $H R$ hazard ratio, $L v$ leucovorin, $O x$ oxaliplatin, $P T X$ paclitaxel, $R R$ risk ratio, $S E$ standard error

two studies were eligible to assess S-1-based versus capecitabine-based therapy [30, 31] , and six studies were eligible to assess $\mathrm{S}-1$ combination therapy versus S-1 monotherapy [21, 32-35]. Searching ClinicalTrials.gov and the American Society of Clinical Oncology and European Society for Medical Oncology libraries provided additional reports of four unpublished phase III studies [11, 12, 14, 15] and two phase II studies [10, 13]. The total number of studies included was 18 (Fig. S1).

There were no major differences in study and patient characteristics among the studies included (Table 1), although one study included patients with diffuse gastric cancer only [11]. For the primary outcome, seven studies were rated as low risk of bias [28-34], whereas 11 studies were rated as unclear risk of bias because of the lack of information on one item (three studies) [12, 21, 35] or two items (three studies) [20, 27] or abstract and insufficient information for risk of bias assessment (five studies) [10, 11, 13-15] (Fig. S2).

\section{S-1-based therapy versus 5-FU- and capecitabine- based therapy}

Eleven studies $(n=3135)$ were included in the metaanalysis: 1636 patients received S-1-based therapy, 1334 patients received 5-FU-based therapy (eight studies) and 165 patients received capecitabine-based therapy (three studies). Nine studies were conducted in Asia $(n=1745)$ and two studies were conducted in Western countries $(n=1372)$ (Table 1$)$. We were able to extract OS and PFS data from ten and six studies. respectively, whereas ORR data were available from all 11 studies.

Compared with 5-FU-based therapy, S-1-based therapy showed no difference in OS (HR 0.92, $95 \%$ CI 0.82-1.03, $P=0.16$ ) and PFS (HR 0.88, 95\% CI 0.73-1.08, $P=0.22$ ), but there was a significant increase in ORR (RR 1.43, $95 \%$ CI 1.05-1.96, $P=0.02$ ) (Fig. 1). No statistically significant subgroup differences were found between Asian and Western patients in terms of OS $(P=0.85)$, PFS $(P=0.55)$ and ORR $(P=0.63)$ (Fig. 2$)$. In the Asian population, S-1-based therapy was superior in terms of ORR compared with 5-FU-based therapy $(P=0.02)$, whereas in the Western population, statistical significance was not reached $(P=0.52)$. No significant heterogeneity was detected for $\mathrm{OS}\left(I^{2}=26 \%, P=0.23\right)$; for both PFS and ORR, heterogeneity was present, with $I^{2}=72 \%(P<0.01)$ and $I^{2}=78 \%(P<0.001)$. 
a Overall Survival

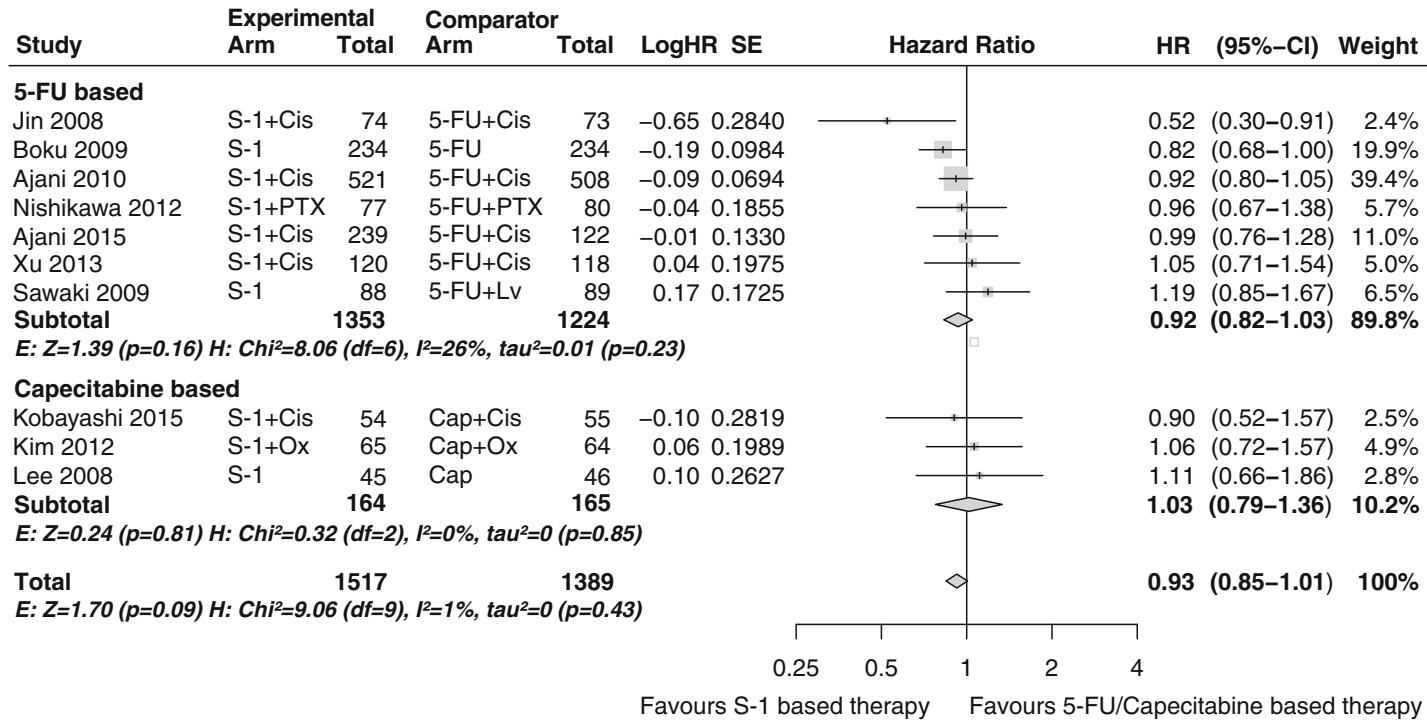

b Progression Free Survival

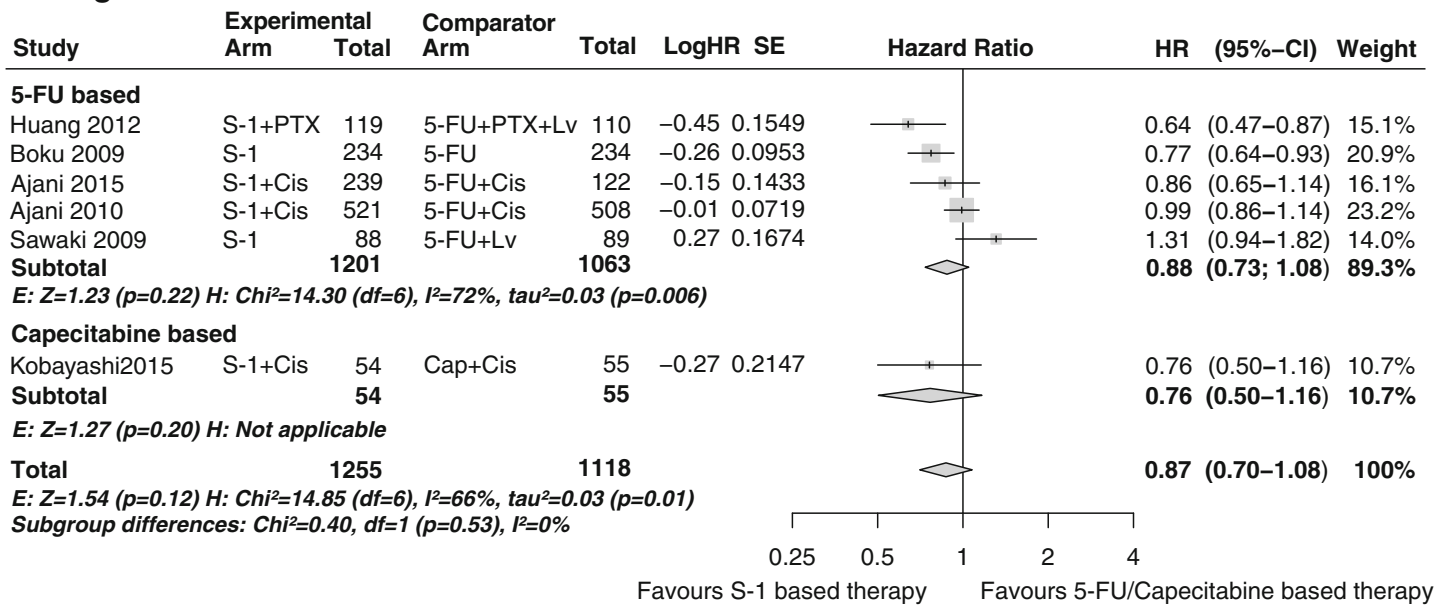

\section{Objective Response Rate}

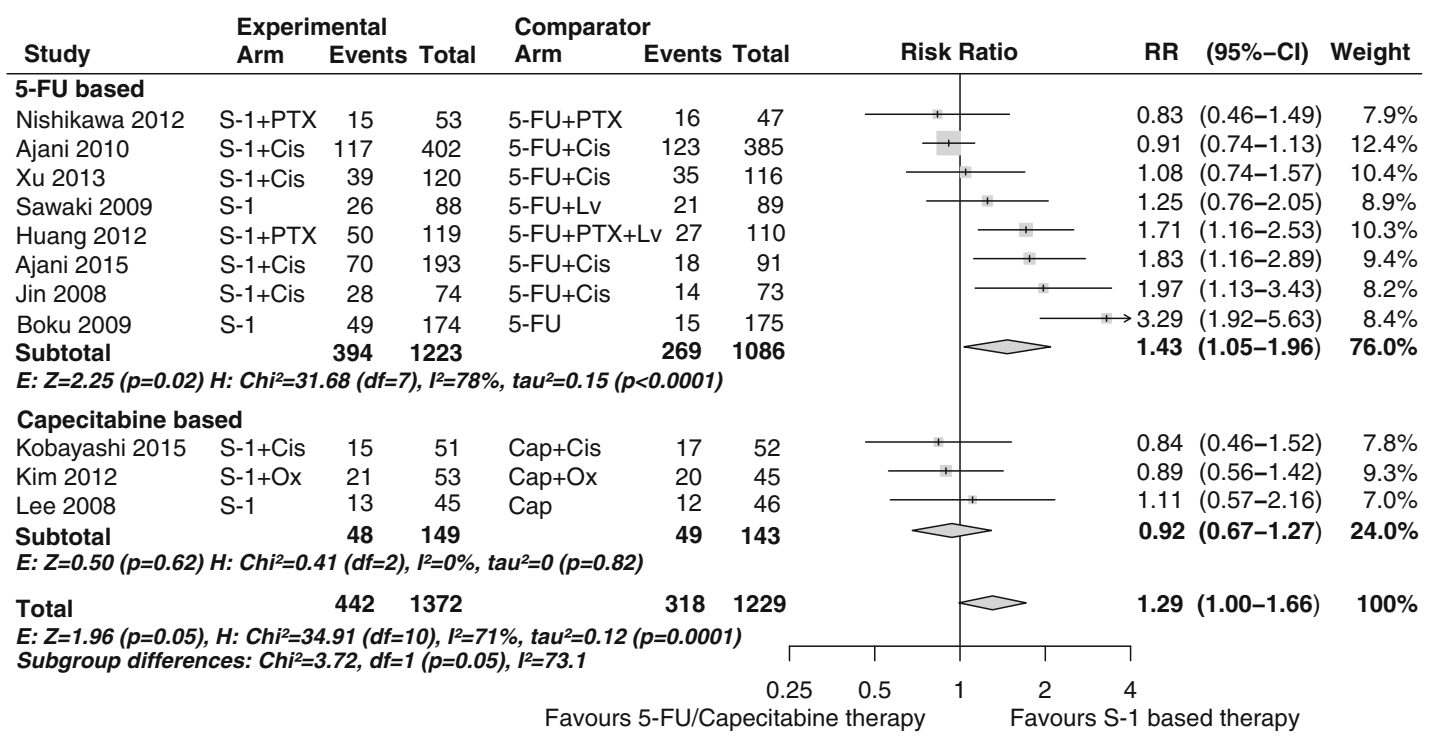


Compared with capecitabine-based therapy, S-1-based therapy showed no difference in OS (HR 1.03, $95 \%$ CI $0.79-1.35, P=0.81$ ), PFS (HR 0.76, $95 \%$ CI $0.50-1.16$, $P=0.20)$ and ORR (RR 0.92, $95 \%$ CI $0.67-1.27$, $P=0.61$ ) (Fig. 2). No statistically significant heterogeneity was detected.

For both comparisons, sensitivity analysis showed that the direction of the overall results was not influenced by omission of studies reported in conference abstracts only, by omission of studies stepwise according to their risk of bias, or by omission of two studies that had leucovorin in the 5-FU arm, which was the case in the studies of Sawaki et al. [15] and Huang et al. [27]. This indicates that the results are robust regarding study quality and concomitant administration of leucovorin (Table S1).

For S-1 compared with 5-FU, data were available for four haematological and 14 non-haematological grade 1-2 AEs and for five haematological and 16 non-haematological grade 3-4 AEs (Table 2). In the Western subgroup, S-1-based therapy showed significantly lower rates of febrile neutropenia, toxicity-related deaths, grade 3-4 stomatitis and mucositis and grade 1-2 diarrhoea, stomatitis and alopecia compared with 5-FU-based therapy. The rates of grade 1-2 neutropenia and hand-foot syndrome were greater with S-1 than with 5-FU.

In the Asian subgroup, S-1-based therapy showed a significantly increased incidence of grade 3-4 fatigue and grade 1-2 abdominal pain but a lower incidence of grade 1-2 neutropenia, nausea and weight loss compared with 5-FU-based therapy. The incidence of febrile neutropenia, serious AEs or toxicity-related deaths was not different between both arms.

For S-1 compared with capecitabine, data were available for four haematological and 13 non-haematological grade 1-2 AEs and for five haematological and 12 non-haematological grade 3-4 AEs (Table 3). Lower rates of grade 3-4 neutropenia and grade 1-2 hand-foot syndrome were found with S-1-based therapy compared with capecitabinebased therapy. The incidence of febrile neutropenia, serious AEs or toxicity-related deaths was not different between both arms.

\section{S-1-based combination therapy versus $S-1$ monotherapy}

For this comparison, eight studies ( $n=1808$ ) were included in the meta-analysis, with 927 and 881 patients in the S-1 combination therapy group and the S-1 monotherapy group, respectively. Four different combination therapies were compared with S-1 monotherapy: S-1 plus cisplatin therapy ( $n=544$ patients, three studies), S-1 plus oxaliplatin therapy ( $n=190$, two studies), S-1 plus taxane therapy ( $n=717$, two studies) and S-1 plus irinotecan
Fig. 2 S-1-based therapy compared with 5-fluorouracil (5-FU)-based therapy for Asian and Western patient subgroups: a overall survival; b progression-free survival; c overall response rate. Cap capecitabine, $C I$ confidence interval, $C i s$ cisplatin, $d f$ degrees of freedom, $E$ effect, $H$ heterogeneity, $H R$ hazard ratio, $L v$ leucovorin, $P T X$ paclitaxel, $R R$ risk ratio, $S E$ standard error

therapy ( $n=404$, two studies). All studies were conducted in Asia: three studies in China, four studies in Japan, and one study in both Japan and Korea (Table 1). We extracted the HRs and $95 \%$ CIs from seven studies for OS and from five studies for PFS. ORRs were available from all eight studies.

The pooled estimates of S-1 combination therapy versus S-1 monotherapy were superior for OS (HR 0.76, $95 \% \mathrm{CI}$ $0.65-0.89, P<0.001$ ), PFS (HR 0.68, $95 \%$ CI 0.56-0.82, $P<0.001)$ and ORR (RR 1.51, $95 \%$ CI 1.32-1.74, $P<0.001$ ) (Fig. 3). Subgroup analyses showed that ORR was significantly better for all four combination therapies and showed no evidence of heterogeneity $\left(I^{2}=0 \%\right.$, $P=0.95)$. However, only $\mathrm{S}-1$ plus oxaliplatin therapy showed significant estimates for both OS and PFS compared with S-1 monotherapy, whereas OS was not significant for S-1 combined with irinotecan, cisplatin or a taxane. PFS was statistically significant for $S-1$ plus taxane therapy, but not for S-1 plus cisplatin therapy or S-1 plus irinotecan therapy.

Heterogeneity was explored in subanalyses and sensitivity analyses (Table S2). For the cisplatin-based and taxane-based subgroup analyses, the non-significant effect might by due to some heterogeneity among the studies (OS $I^{2}=45.0 \%, P=0.08$; PFS $\left.I^{2}=44 \%, P=0.11\right)$. When studies were stratified according to region, a significant subgroup difference between Chinese studies and Japanese studies was found in OS $(P<0.005)$. No subgroup differences for region were found in PFS $(P=0.38)$ and ORR $(P=0.88)$. Furthermore, no significant fluctuations in the overall results were detected with sensitivity analysis according to study quality and concomitant administration of leucovorin, which was the case with the comparison of S-1 plus cisplatin therapy with S-1 plus leucovorin therapy in the study of Yamaguchi et al. [13].

Data were available for four haematological and 12 nonhaematological grade 1-2 AEs and for five haematological and 11 non-haematological grade 3-4 AEs. Compared with S-1 monotherapy, S-1-based doublets were associated with an increased rate of grade 3-4 neutropenia, leucopenia and stomatitis and with an increased rate of grade 1-2 leucopenia, anaemia, thrombocytopenia, lymphocytopenia, anorexia, fatigue and alopecia (Table S3).

To identify subgroups that may benefit most from $\mathrm{S}-1$ combination therapy compared with S-1 monotherapy, three large phase III Japanese studies $(n=1248)$ reporting a stratified analysis for OS could be used (Fig. 4) [32, 33]. 


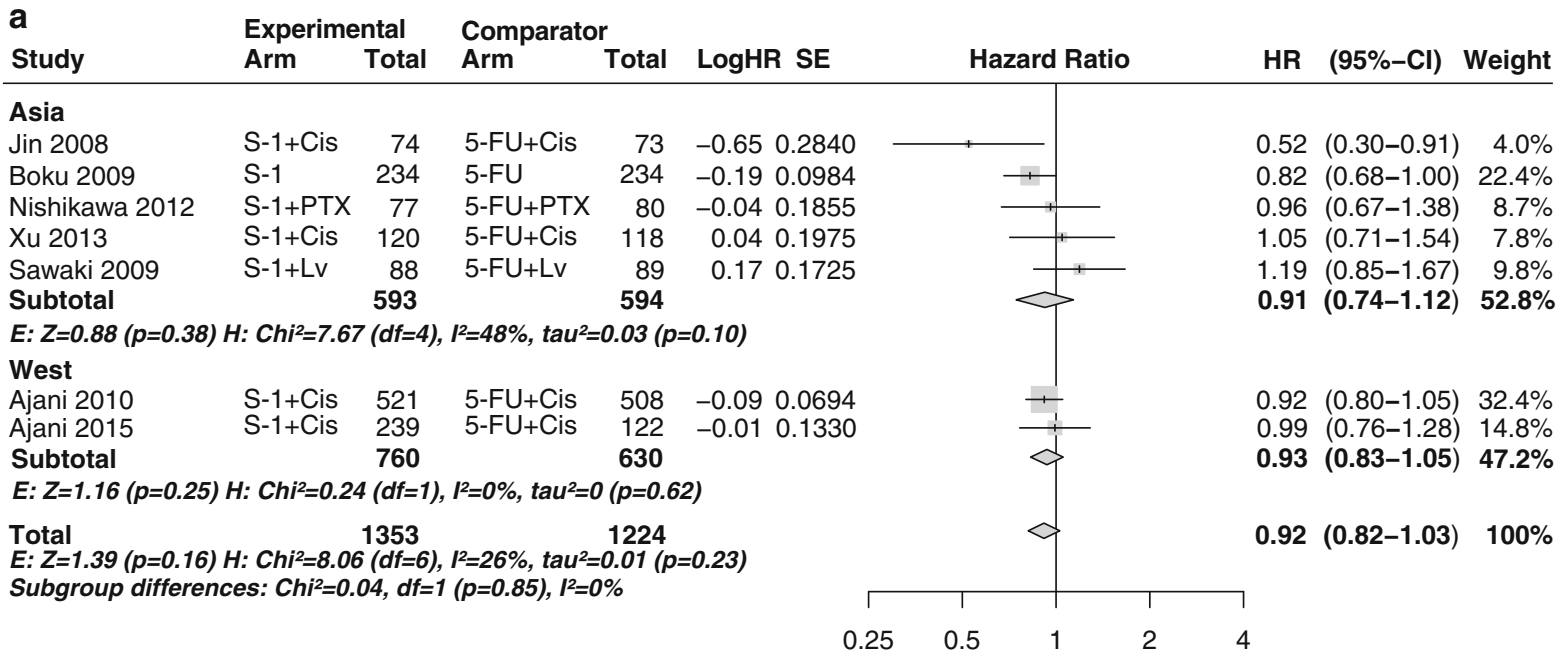

Favours S-1 based therapy Favours 5-FU based therapy

b

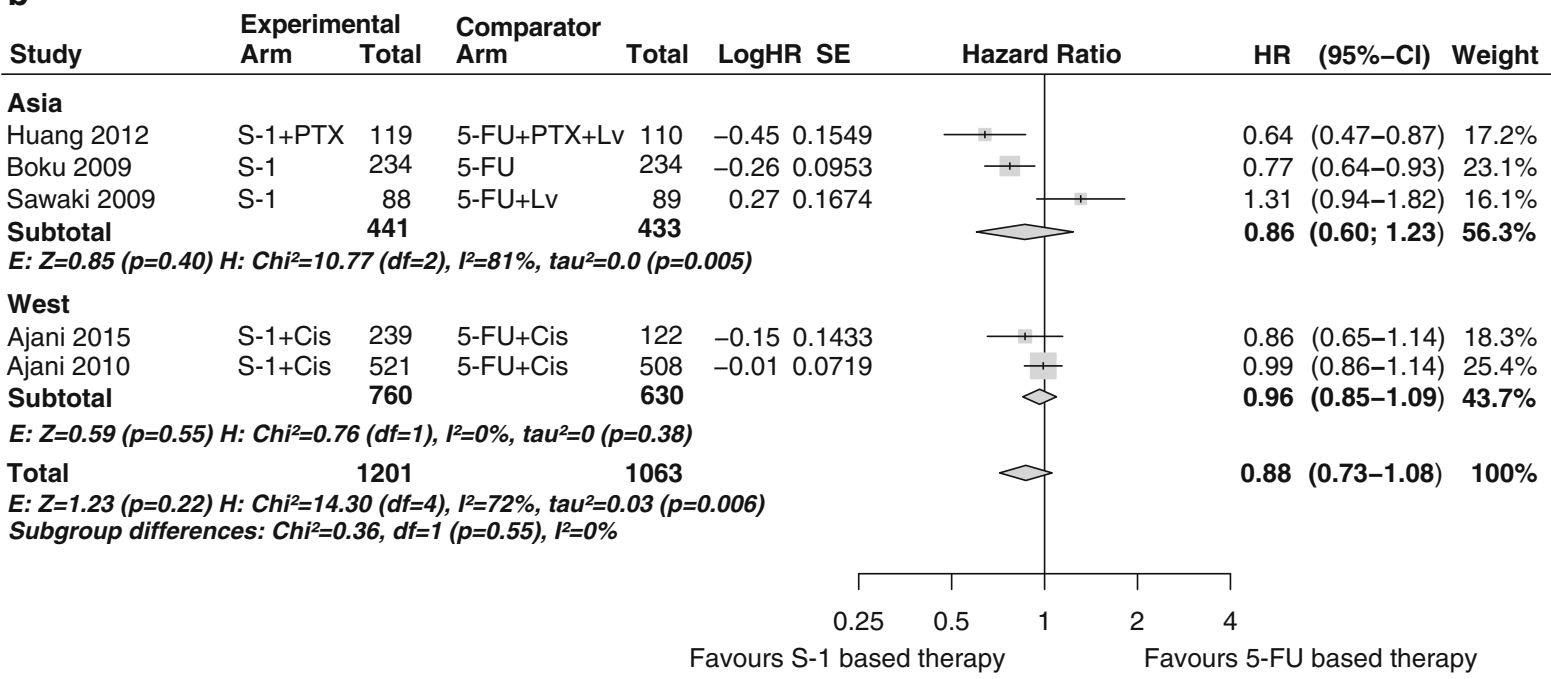

C

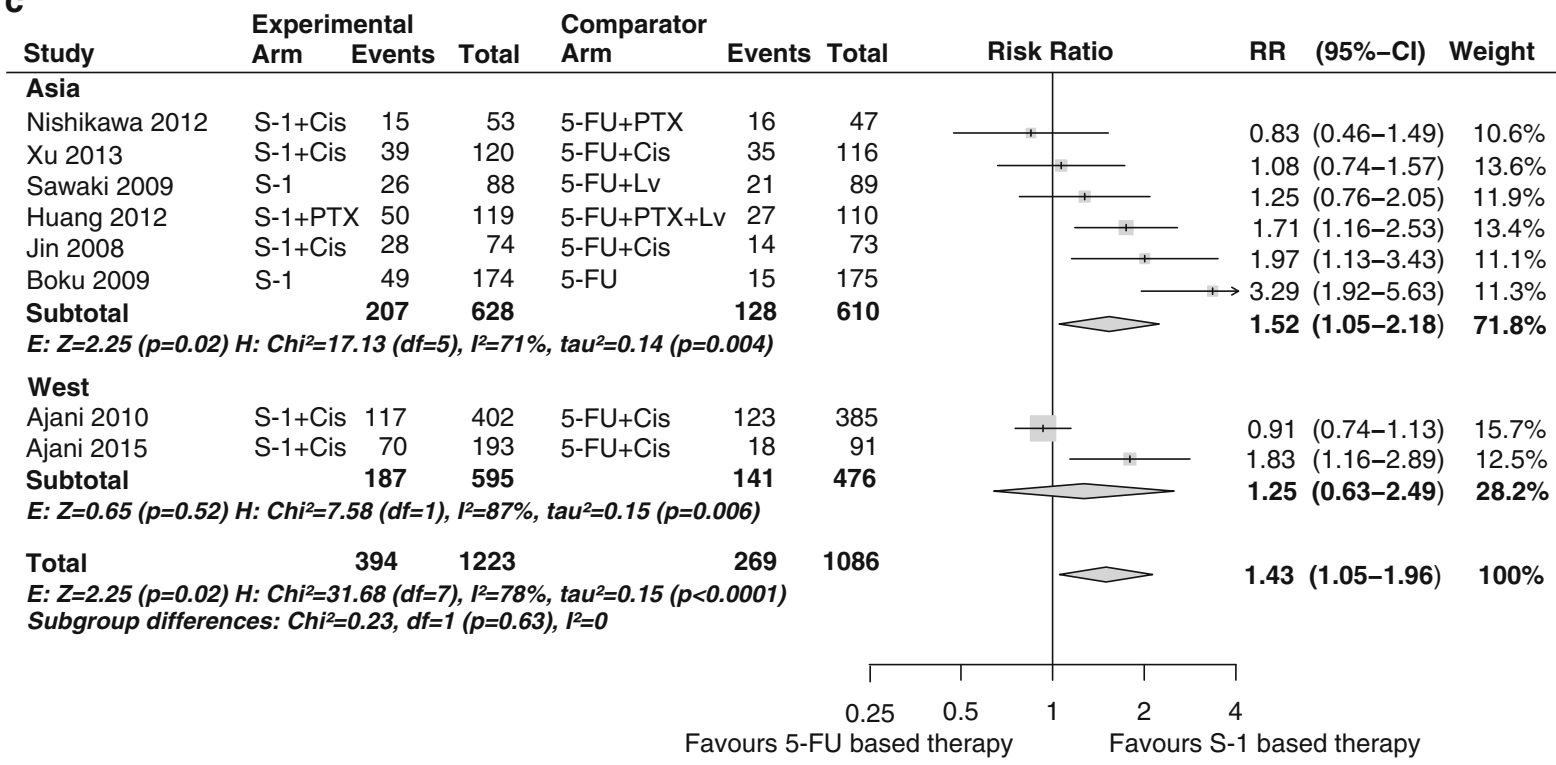




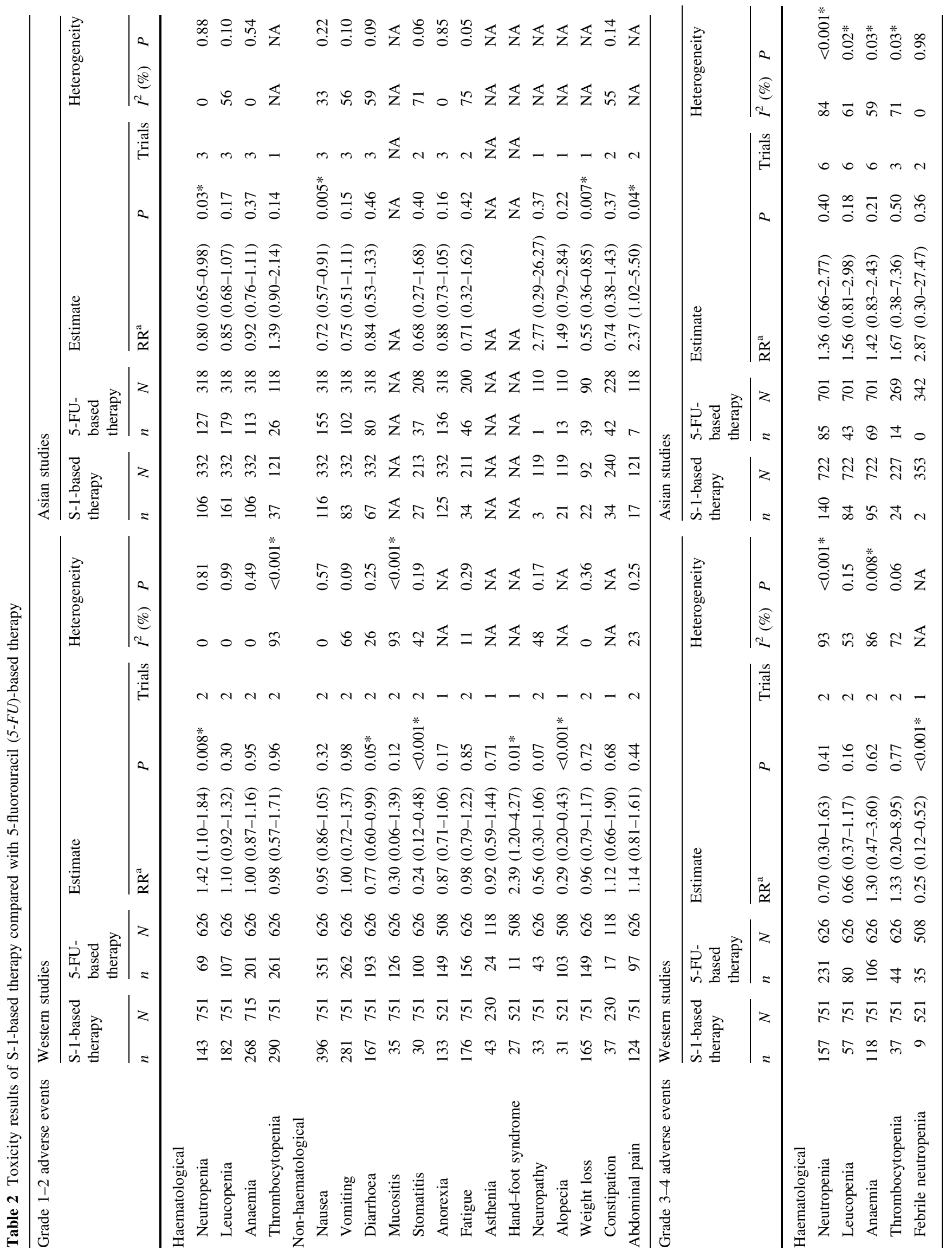




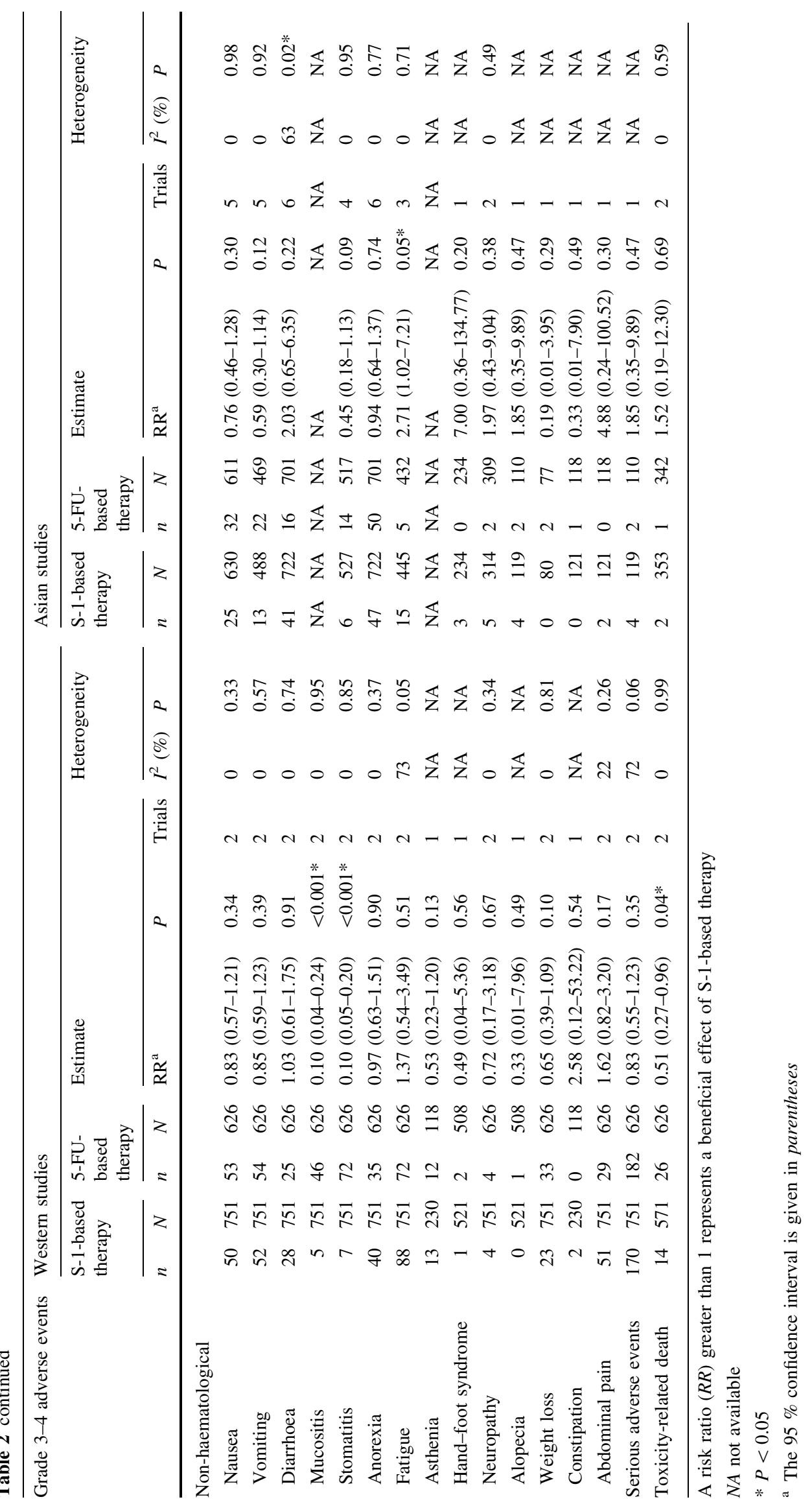




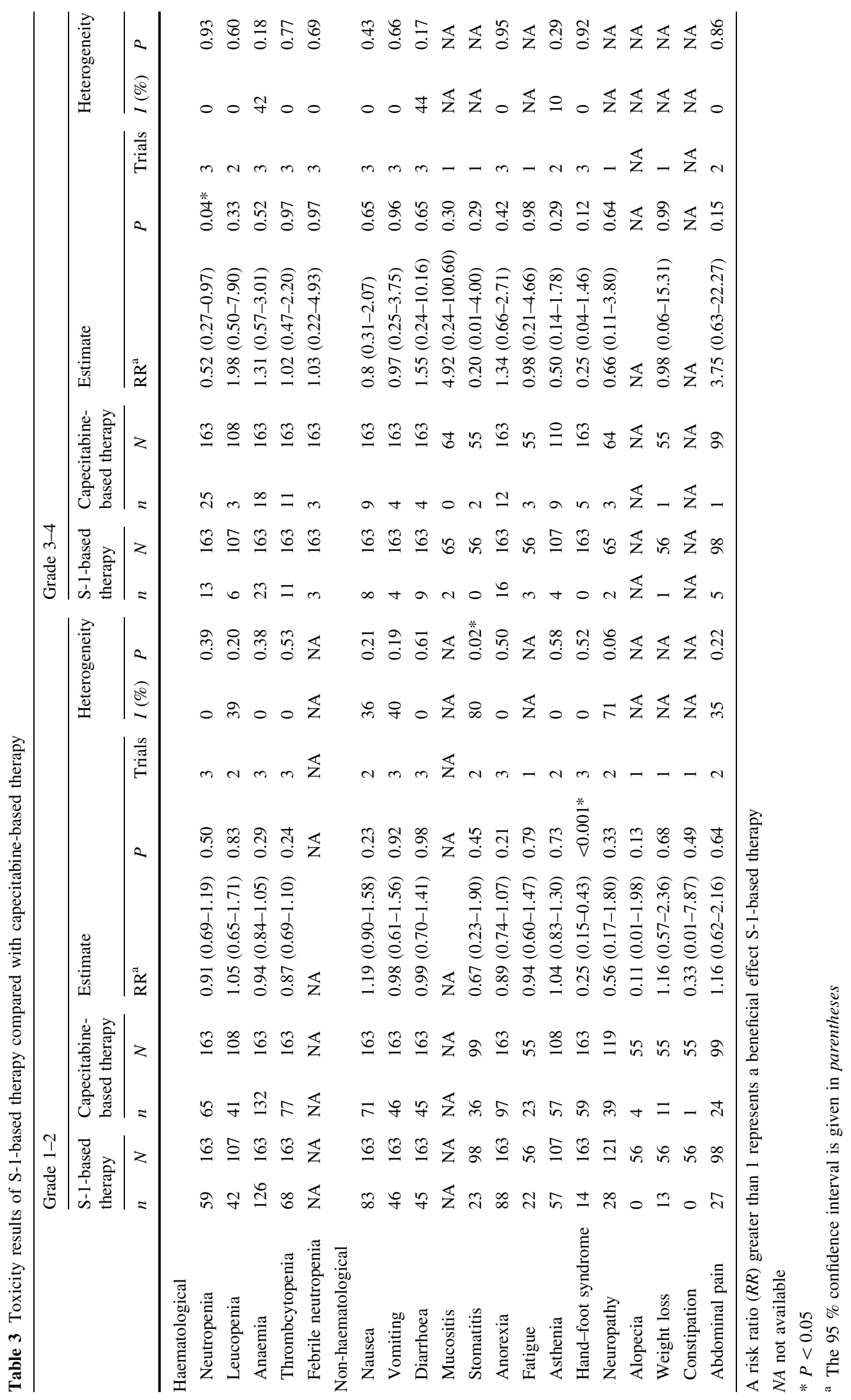


Fig. 3 S-1-based combination therapy compared with S-1 monotherapy: a overall survival; b progression-free survival; c overall response rate. $C I$ confidence interval, $C i$ s cisplatin, $d f$ degrees of freedom, $D T X$ docetaxel, $E$ effect, $H$ heterogeneity, $H R$ hazard ratio, IRI irinotecan, $L v$ leucovorin, $N A$ not available, $O x$ oxaliplatin, $P T X$ paclitaxel, $R R$ risk ratio, $S E$ standard error

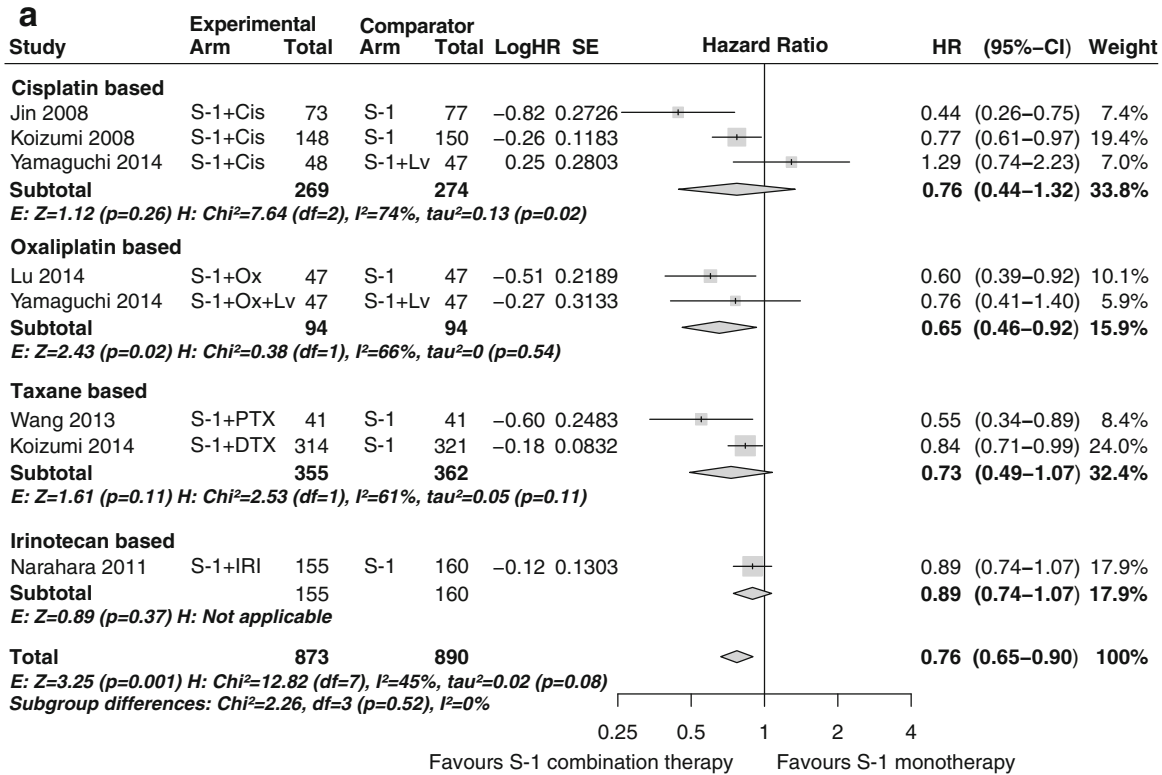

b

Experimental Comparator

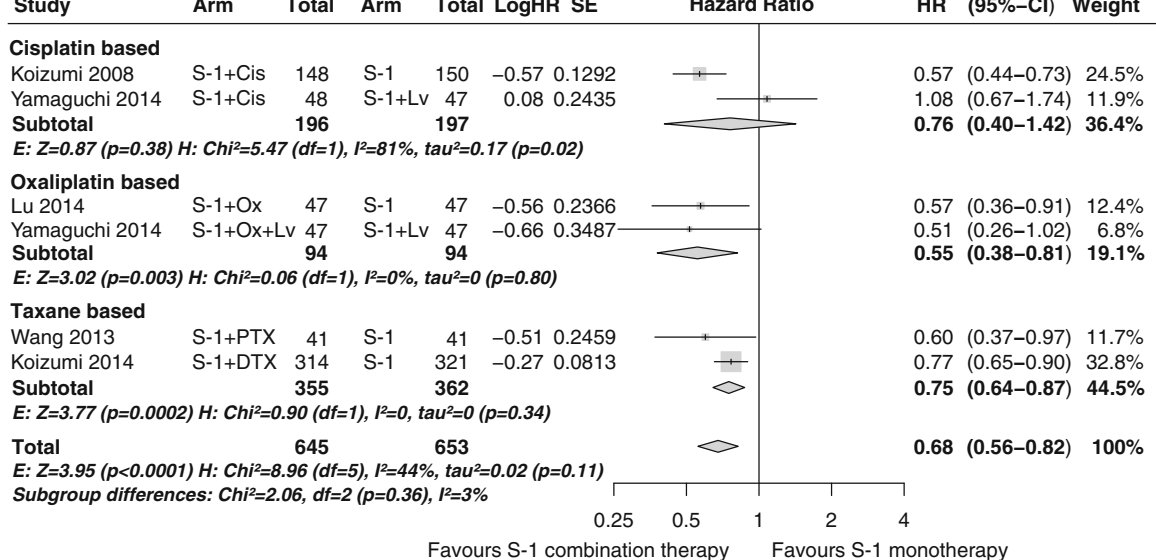

\begin{tabular}{|c|c|c|c|c|c|c|c|c|c|c|c|}
\hline \multirow{3}{*}{$\begin{array}{l}\text { C } \\
\text { Study } \\
\text { Cisplatin based }\end{array}$} & \multicolumn{3}{|c|}{ Experimental } & \multicolumn{2}{|c|}{ Comparator } & \multirow{2}{*}{ Total } & \multirow{2}{*}{\multicolumn{2}{|c|}{ Risk Ratio }} & \multirow[b]{2}{*}{ RR } & \multirow[b]{2}{*}{$(95 \%-\mathrm{Cl})$} & \multirow[b]{2}{*}{ Weight } \\
\hline & Arm $E$ & & Total & Arm $\mathrm{E}$ & & & & & & & \\
\hline & & & & & & & & & & & \\
\hline Jin 2008 & $\mathrm{~S}-1+\mathrm{Cis}$ & 28 & 74 & S-1 & 19 & 77 & & & 1.53 & $(0.94-2.50)$ & $8.1 \%$ \\
\hline Koizumi 2008 & $\mathrm{~S}-1+\mathrm{Cis}$ & 47 & 87 & S-1 & 33 & 106 & & $\longrightarrow$ & 1.74 & $(1.23-2.45)$ & $16.4 \%$ \\
\hline Yamaguchi 2014 & $\mathrm{~S}-1+\mathrm{Cis}$ & 22 & 47 & $S-1+L v$ & 20 & 47 & & 1 & 1.10 & $(0.70-1.73)$ & $9.5 \%$ \\
\hline Subtotal & & 97 & 208 & & 72 & 230 & & $\infty$ & 1.44 & $(1.10-1.89)$ & $34.0 \%$ \\
\hline \multicolumn{12}{|c|}{$E: Z=2.79(p=0.005) H: C h i^{2}=2.51(d f=2), l^{2}=20 \%, \operatorname{tau}^{2}=0.01(p=0.28)$} \\
\hline \multicolumn{12}{|l|}{ Oxaliplatin based } \\
\hline Lu 2014 & $\mathrm{~S}-1+\mathrm{Ox}$ & 24 & 47 & S-1 & 13 & 47 & & 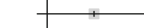 & 1.85 & $(1.08-3.17)$ & $6.6 \%$ \\
\hline Yamaguchi 2014 & $\mathrm{~S}-1+\mathrm{Ox}+\mathrm{L}$ & Lv 31 & 48 & S-1+Lv & 20 & 47 & & 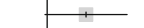 & 1.52 & $(1.02-2.25)$ & $12.5 \%$ \\
\hline Subtotal & & 55 & 95 & & 33 & 94 & & 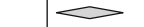 & 1.62 & $(1.18-2.23)$ & $19.1 \%$ \\
\hline \multicolumn{12}{|c|}{$E: Z=2.99(p=0.003) H: C h i^{2}=0.34(d f=1), I^{2}=0 \%, \operatorname{tau}^{2}=0(p=0.56)$} \\
\hline \multicolumn{12}{|c|}{ Taxane based } \\
\hline Koizumi 2014 & $\mathrm{~S}-1+\mathrm{PTX}$ & 92 & 237 & S-1 & 65 & 243 & & $\longrightarrow$ & 1.45 & $(1.12-1.89)$ & $28.0 \%$ \\
\hline Wang 2013 & S-1+DTX & 19 & 41 & S-1 & 10 & 41 & & & 1.90 & $(1.01-3.57)$ & $4.8 \%$ \\
\hline Subtotal & & 111 & 278 & & 75 & 284 & & $\infty$ & 1.51 & (1.19-1.92) & $32.8 \%$ \\
\hline \multicolumn{12}{|c|}{$E: Z=3.33(p=0.0009) H: C h i^{2}=0.60(d f=1), l^{2}=0 \%, \operatorname{tau}^{2}=0(p=0.44)$} \\
\hline \multicolumn{12}{|l|}{ Irinotecan based } \\
\hline Komatsu 2011 & S-1+|R| & 10 & 36 & S-1 & 7 & 32 & & $\longrightarrow$ & 1.27 & $(0.55-2.94)$ & $2.7 \%$ \\
\hline Narahara 2011 & S-1+|R| & 39 & 94 & S-1 & 25 & 93 & & 1 & 1.54 & $(1.02-2.33)$ & $11.3 \%$ \\
\hline Subtotal & & 49 & 130 & & 32 & 125 & & 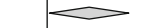 & 1.49 & (1.03-2.15) & $14.1 \%$ \\
\hline \multicolumn{12}{|c|}{$E: Z=2.10(p=0.04) H: C h i^{2}=0.17(d f=1), l^{2}=0 \%, \operatorname{tau}^{2}=0(p=0.68)$} \\
\hline Total & & 312 & 711 & & 212 & 733 & & $\infty$ & 1.51 & $(1.32-1.74)$ & $100 \%$ \\
\hline \multirow{2}{*}{\multicolumn{12}{|c|}{$\begin{array}{l}E: Z=5.90(p<0.00001) H: C h i^{2}=3.38(d f=8), I^{2}=0 \%, \operatorname{tau}^{2}=0(p=0.87) \\
\text { Subgroup differences: } \mathrm{Ch}^{2}=0.24, d f=3(p=0.97), I^{2}=0\end{array}$}} \\
\hline & & & & & & & & & 7 & & \\
\hline & & & & & & 0.25 & 0.5 & 1 & 4 & & \\
\hline
\end{tabular}


The pooled effect size for these three studies was HR 0.82 (95\% CI 0.72-0.93). A trend toward significant subgroup differences in favour of S-1 combination therapy was found in favour of patients with diffuse-type histological features compared with patients with intestinal-type histological features $(P=0.06$; HR $<0.80)$ and patients with measurable disease compared with patients with non-measurable disease $(P=0.06 ; \quad \mathrm{HR}<0.80)$. Furthermore, subgroups with peritoneal metastases showed a non-significant but clinically relevant HR ( 0.80 or less) in favour of S-1 combination therapy. No other potential predictive factors were identified.

\section{Publication bias}

Funnel plots did not show significant asymmetry and Egger's test was not significant for S-1-based therapy versus 5-FU/capecitabine-based therapy in terms of OS $(P=0.75)$, PFS $(P=0.82)$, and ORR $(P=0.73)$ and for S-1-based combination therapy versus S-1 monotherapy in terms of OS $(P=0.08)$, PFS $(P=0.71)$ and ORR $(P=0.96)$ (Figure S3).

\section{Discussion}

Previous meta-analyses have suggested that 5-FU may be replaced by $\mathrm{S}-1$ in first-line therapy for advanced gastric cancer because of a survival benefit in favour of S-1 [3, 4]. Our updated meta-analysis does not confirm this finding. Although a higher ORR was observed for S-1-based therapy versus 5-FU-based therapy, OS and PFS were not significantly prolonged. The pooled OS and PFS effect sizes of the two recently conducted Western studies, the FLAGS and DIGEST trials, were comparable to the pooled OS and PFS effect sizes of all Asian studies. This suggests that S-1 may have similar efficacy in both Western and Asian patients. However, in Western patients S-1-based therapy did have clear clinically relevant advantages in terms of the toxicity profile over 5-FU-based therapynamely, lower rates of febrile neutropenia, toxicity-relateddeaths and grade 3-4 mucositis and stomatitis, whereas the toxicity profiles of S-1 and 5-FU in Asian patients showed no clinically relevant differences, except a higher rate of grade 3-4 fatigue and lower rates of grade 1-2 neutropenia and nausea. This indicates that S-1 is well tolerated in Western patients with its current dosing as used in the FLAGS and DIGEST trials.

Also, S-1 was not more effective than capecitabine in Asian patients. In the West, it has been suggested that capecitabine may be replaced by S-1 in the case of handfoot syndrome. This meta-analysis shows that the incidence of grade 1-2 hand-foot syndrome was significantly lower
Fig. 4 Stratified overall survival (OS) results for S-1 combination therapy versus S-1 monotherapy. Forest plot of OS results for S-1based combination therapy versus S-1 monotherapy stratified per patient subgroup. For target tumour more than three studies are shown because these studies included only patients with measurable lesions. Pooled sample sizes are stated for S-1 combination therapy and S-1 monotherapy groups if separate sample sizes were not available in the study report. CI confidence interval, Cis cisplatin, $d f$ degrees of freedom, DTX docetaxel, $E$ effect, ECOG Eastern Cooperative Oncology Group performance status, $H$ heterogeneity, $H R$ hazard ratio, IRI irinotecan, $O x$ oxaliplatin, $P T X$ paclitaxel

with S-1 than with capecitabine. We stress that hand-foot syndrome specifically can have a severe impact on quality of life, because capacitabine is usually given for a longer time. Moreover, in a previous review which also included studies in metastatic colorectal cancer, a significantly lower rate of grade 3-4 hand-foot syndrome was observed for S-1 $(0.3 \%)$ compared with capecitabine (3.1\%); $P<0.001$ [7]. Also, in our meta-analysis there were fewer observations of grade 3-4 hand-foot syndrome with S-1 $(0.0 \%)$ versus capecitabine $(3.1 \%)$, but the numbers were too low to reach statistical significance. Because all capecitabine studies were conducted in Asia, we should interpret our findings with caution for Western populations..

This is the first meta-analysis to examine the differential efficacy of combination therapy and monotherapy in patients with different baseline factors and can aid in clinical decision making. Overall, we showed that S-1 combination therapy is more efficacious than $\mathrm{S}-1$ monotherapy. Importantly, our meta-analysis of stratified data from the three largest studies suggests that patients with disease characteristics associated with poor prognosis, such as non-measurable lesion, diffuse-type histological features and peritoneal metastasis, may have increased benefit from combination therapy.

The pooled result for the OS benefit of taxane combinations was not convincing because of heterogeneity. However, the HR (0.73) may be considered clinically meaningful and the PFS was significantly prolonged. Improvement of PFS may also be an important finding, because PFS is less prone to the influence of second-line therapy than OS. More grade 1-2 and grade 3-4 haematological toxicity as well as gastrointestinal toxicity occurred with combination therapy compared with monotherapy, which was in line with other combination chemotherapy regimens including a fluoropyrimidine combined with platinum compounds [36, 37], taxanes [38, 39] or irinotecan [37, 40].

Our study has some limitations. First, we did not take specific dosing regimens into account, which could have impacted our results. With pooled data analyses, including meta-analysis, it is often not possible to investigate to what extent dose differences may have influenced the results of 


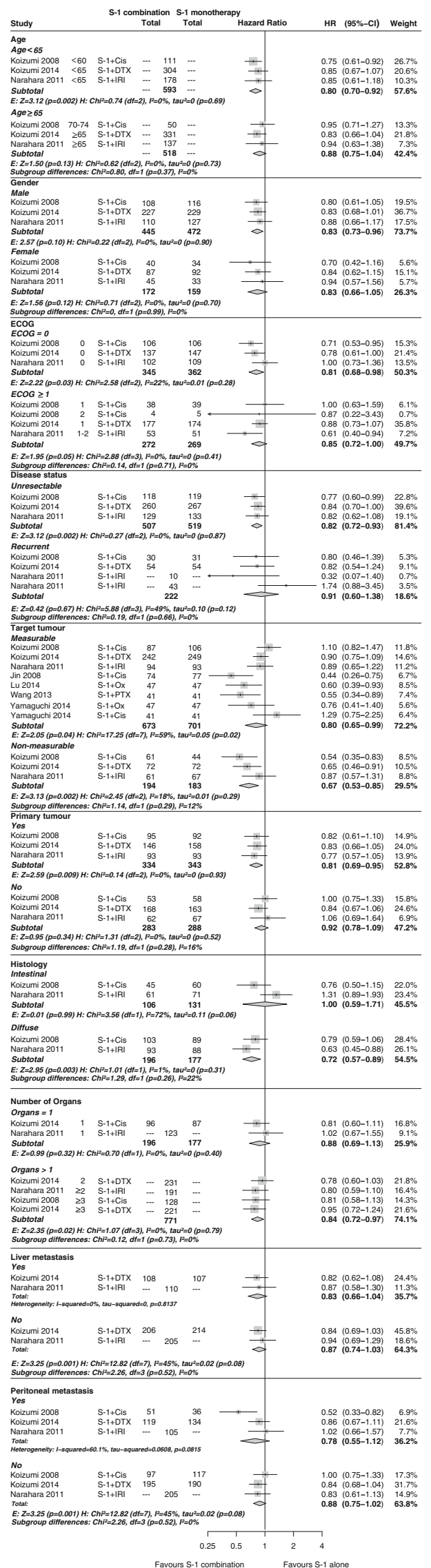

the meta-analysis. Also, in some studies, leucovorin was added to fluoropyrimidine therapy. Leucovorin increases the intracellular pool of 5,10-methylenetetrahydrofolate, thereby enhancing thymidylate synthase inhibition by fluorodeoxyuridine monophosphate [41]. This mechanism of action implies that leucovorin should be regarded not as an additional cytotoxic agent but rather as a modulator of fluoropyrimidine efficacy and toxicity. We conducted sensitivity analyses in which we omitted the studies in which leucovorin was concomitantly administrated with one of the S-1 or 5-FU regimens. This did not affect the pooled effect sizes of all comparisons. Furthermore, most of the fluoropyrimidine dosing regimens of the studies included in our review were similar. Especially the dosing of S-1 is fairly constant among different studies.

A second limitation is that the heterogeneity due to the difference in OS effect size in the Chinese subgroup and Japanese subgroup may somewhat complicate the interpretation of the S-1 combination therapy versus S-1 monotherapy analysis. Two of the Chinese studies were single-centre studies, whereas all Japanese studies were multicentre studies and therefore may have higher quality. However, the sensitivity analysis according to the risk of bias did not suggest major fluctuations in results. Whether there is a real difference in efficacy for combination therapy between Chinese and Japanese populations or whether this is purely a methodological issue remains unclear and should be addressed in larger and more qualitatively sound studies with Chinese patients.

In summary, S-1-based therapy showed no difference in survival compared with 5-FU- and capecitabine-based therapy but has a higher ORR compared with 5-FU-based therapy. In terms of clinical relevance, the toxicity profile of S-1 compared with 5-FU was clearly more advantageous in Western patients. Also, S-1 showed a better toxicity profile compared with capecitabine, with a lower incidence of hand-foot syndrome. In general, S-1 combination therapy is superior to S-1 monotherapy in terms of efficacy, and patients with poor prognosis disease characteristics may benefit most from S-1 combination therapy, although S-1 combinations were more toxic than S-1 alone. Our findings suggest that $\mathrm{S}-1$-based regimens are effective and tolerable as first-line treatment of advanced gastric cancer in both Asian and Western countries.

Acknowledgment We thank Sam ter Veer for the graphical design of the figures.

Author contributions The literature search was performed by Emil ter Veer, Nadia Haj Mohammad and Mary Samaan. Quality assessment was done by Emil ter Veer, Nadia Haj Mohammad and Hanneke W.M. van Laarhoven. Data extraction was done by Emil ter Veer, Lok Lam Ngai and Nadia Haj Mohammad. Statistical analysis was performed by Paul Lodder, Emil ter Veer and Lok Lam Ngai. The manuscript was written by Emil ter Veer, Nadia Haj Mohammad, 
Hanneke W.M. van Laarhoven and Martijn G.H. van Oijen. Martijn G.H. van Oijen and Hanneke W.M. van Laarhoven supervised the study. All authors gave final approval for submission of the manuscript.

\section{Compliance with ethical standards}

Funding There is no funding source to declare.

Conflict of interest Hanneke W.M. van Laarhoven is consultant of Nordic and has received research funding from Nordic.

Ethics statement In all studies that were included in this systematic review it was declared that all procedures followed were in accordance with the ethical standards of the responsible committee on human experimentation (institutional and national) and with the Helsinki Declaration of 1964 and later versions. Also, in all studies it was declared that informed consent or substitute for it was obtained from all patients for their being included in the studies.

Open Access This article is distributed under the terms of the Creative Commons Attribution 4.0 International License (http://crea tivecommons.org/licenses/by/4.0/), which permits unrestricted use, distribution, and reproduction in any medium, provided you give appropriate credit to the original author(s) and the source, provide a link to the Creative Commons license, and indicate if changes were made.

\section{References}

1. Waddell T, Verheij M, Allum W, Cunningham D, Cervantes A, Arnold D. Gastric cancer: ESMO-ESSO-ESTRO clinical practice guidelines for diagnosis, treatment and follow-up. Eur J Surg Oncol. 2014;40(5):584-91. doi:10.1016/j.ejso.2013.09.020.

2. Ajani JA, Bentrem DJ, Besh S, D'Amico TA, Das P, Denlinger C, et al. Gastric cancer, version 2.2013. J Natl Compr Canc Netw. 2013;11(5):531-46.

3. Yang J, Zhou Y, Min K, Yao Q, Xu CN. S-1-based vs non-S-1based chemotherapy in advanced gastric cancer: a meta-analysis. World J Gastroenterol. 2014;20(33):11886-93.

4. Li DH, Pan ZK, Ye F, An HX, Wu JX. S-1-based versus 5-FUbased chemotherapy as first-line treatment in advanced gastric cancer: a meta-analysis of randomized controlled trials. Tumour Biol. 2014. doi:10.1007/s13277-014-2099-2.

5. Huang J, Cao Y, Wu L, Liao C, He Y, Gao F. S-1-based therapy versus 5-FU-based therapy in advanced gastric cancer: a metaanalysis. Med Oncol. 2011;28(4):1004-11.

6. Cao C, Zhang X, Kuang M, Gu D, He M, Chen J, et al. Survival benefit from S-1 as compared to fluorouracil in Asian patients with advanced gastrointestinal cancer: a meta-analysis. Cancer Sci. 2014;105(8):1008-14. doi:10.1111/cas.12465.

7. Zhang X, Cao C, Zhang Q, Chen Y, Gu D, Shen Y, et al. Comparison of the efficacy and safety of S-1-based and capecitabine-based regimens in gastrointestinal cancer: a meta-analysis. PLoS One. 2014;9(1):e84230.

8. He MM, Wu WJ, Wang F, Wang ZQ, Zhang DS, Luo HY, et al. S-1-based chemotherapy versus capecitabine-based chemotherapy as first-line treatment for advanced gastric carcinoma: a meta-analysis. PLoS One. 2013;8(12):e82798. doi:10.1371/jour nal.pone.0082798.

9. He AB, Peng XL, Song J, Zhang JX, Dong WG, Luo RF, et al. Efficacy of S-1 vs capecitabine for the treatment of gastric cancer: a meta-analysis. World J Gastroenterol. 2015;21(14):4358-64. doi:10.3748/wjg.v21.i14.4358.

10. Kobayashi M, Tsuburaya A, Nishikawa K, Kawada J, Namikawa T, Fukushima R. A randomized phase II trial of capecitabine plus cisplatin (XP) versus S-1 plus cisplatin (SP) as a first-line treatment for advanced gastric cancer: XP ascertainment versus SP randomized PII trial (XParTS II). J Clin Oncol. 2015;33(3 Suppl):105.

11. Ajani JA, Abramov M, Bondar V, Bondarenko I, Shparyk YV, Gorbunova V, et al. Untreated metastatic diffuse gastric adenocarcinoma (DGAC): randomized phase III study of S-1 and cisplatin vs. 5-FU and cisplatin (the DIGEST trial). J Clin Oncol. 2015;33(15 Suppl):4015.

12. Jin M, Lu H, Li J, Shen L, Chen Z, Shi Y, et al. Randomized 3 -armed phase III study of S-1 monotherapy versus S-1/CDDP (SP) versus 5-FU/CDDP (FP) in patients (pts) with advanced gastric cancer (AGC): SC-101 study. J Clin Oncol. 2008;26(15 Suppl):4533.

13. Yamaguchi K, Hironaka S, Sugimoto N, Moriwaki T, Komatsu $\mathrm{Y}$, Nishina T, et al. Randomized phase II study of S-1 plus oral leucovorin (SL) versus SL plus oxaliplatin (SOL) versus S-1 plus cisplatin (SP) in patients with advanced gastric cancer. Ann Oncol. 2014;25(Suppl 4):iv210-253. doi:10.1093/annonc/ mdu334.

14. Xu RH, Sun GP, Lu HS, Peng LY, Xu JM, Zhong MZ, et al. A phase III study of S-1 plus cisplatin versus fluorouracil plus cisplatin in patients with advanced gastric or gastroesophageal junction adenocarcinoma. J Clin Oncol. 2013;31:4025.

15. Sawaki A, Yamaguchi K, Nabeya Y, Sakai Y, Osanai H, Denda $\mathrm{T}$, et al. 5-FU/1-LV (RPMI) versus $\mathrm{S}-1$ as first-line therapy in patients with advanced gastric cancer: a randomized phase III non-inferiority trial (ISO-5FU10 Study Group trial). EJC Suppl. 2009;7(2):364.

16. Ajani JA, Rodriguez W, Bodoky G, Moiseyenko V, Lichinitser $\mathrm{M}$, Gorbunova V, et al. Multicenter phase III comparison of cisplatin/S-1 with cisplatin/infusional fluorouracil in advanced gastric or gastroesophageal adenocarcinoma study: the FLAGS trial. J Clin Oncol. 2010;28(9):1547-53. doi:10.1200/JCO.2009. 25.4706.

17. Wagner AD, Unverzagt S, Grothe W, Kleber G, Grothey A, Haerting J, et al. Chemotherapy for advanced gastric cancer. Cochrane Database Syst Rev. 2010;(3):CD004064. doi:10.1002/ 14651858.CD004064.pub3.

18. Liu GF, Tang D, Li P, Wang S, Xu YX, Long AH, et al. S-1based combination therapy vs S-1 monotherapy in advanced gastric cancer: a meta-analysis. World $\mathbf{J}$ Gastroenterol. 2014;20(1):310-8.

19. Wu JR, Tang WZ, Chen X, Xie YT, Chen SY, Peng QL, et al. S-1-based therapy versus S-1 monotherapy in advanced gastric cancer: a meta-analysis. Tumour Biol. 2014;35(4):3283-93. doi:10.1007/s13277-013-1429-0.

20. Koizumi W, Kim YH, Fujii M, Kim HK, Imamura H, Lee KH, et al. Addition of docetaxel to $\mathrm{S}-1$ without platinum prolongs survival of patients with advanced gastric cancer: a randomized study (START). J Cancer Res Clin Oncol. 2014;140(2):319-28. doi:10.1007/s00432-013-1563-5.

21. Lu Y, Liu Z, Zhang J. S-1 plus oxaliplatin vs. S-1 as first-line treatment in patients with previously untreated advanced gastric cancer: a randomized phase II study. J Chemother. 2014;26(3): 159-64.

22. Möbius TWD. Metagen: inference in meta-analysis and meta regression. http://cran.r-project.org/web/packages/metagen/index. html (2014).

23. Tierney JF, Stewart LA, Ghersi D, Burdett S, Sydes MR. Practical methods for incorporating summary time-to-event data into meta-analysis. Trials. 2007;8:16. doi:10.1186/1745-6215-8-16. 
24. Ellis LM, Bernstein DS, Voest EE, Berlin JD, Sargent D, Cortazar P, et al. American Society of Clinical Oncology perspective: raising the bar for clinical trials by defining clinically meaningful outcomes. J Clin Oncol. 2014;32(12):1277-80. doi:10.1200/jco. 2013.53.8009.

25. Sterne JA, Egger M. Funnel plots for detecting bias in metaanalysis: guidelines on choice of axis. J Clin Epidemiol. 2011;54(10):1046-55.

26. Ajani JA, Rodriguez W, Bodoky G, Moiseyenko V, Lichinitser $\mathrm{M}$, Gorbunova V, et al. Multicenter phase III comparison of cisplatin/S-1 with cisplatin/infusional fluorouracil in advanced gastric or gastroesophageal adenocarcinoma study: the FLAGS trial. J Clin Oncol. 2010;28(9):1547-53. doi:10.1200/JCO.2009. 25.4706.

27. Huang D, Ba Y, Xiong J, Xu N, Yan Z, Zhuang Z, et al. A multicentre randomised trial comparing weekly paclitaxel $+\mathrm{S}-1$ with weekly paclitaxel +5 -fluorouracil for patients with advanced gastric cancer. Eur J Cancer. 2013;49(14):2995-3002.

28. Nishikawa K, Morita S, Matsui T, Kobayashi M, Takeuchi Y, Takahashi I, et al. A randomized phase-II trial comparing sequential and concurrent paclitaxel with oral or parenteral fluorinated pyrimidines for advanced or metastatic gastric cancer. Gastric Cancer. 2012;15(4):363-9. doi:10.1007/s10120-011-0124-3.

29. Boku N, Yamamoto S, Fukuda H, Shirao K, Doi T, Sawaki A, et al. Fluorouracil versus combination of irinotecan plus cisplatin versus S-1 in metastatic gastric cancer: a randomised phase 3 study. Lancet Oncol. 2009;10(11):1063-69. doi:10.1016/s14702045(09)70259-1.

30. Lee JL, Kang YK, Kang HJ, Lee KH, Zang DY, Ryoo BY, et al. A randomised multicentre phase II trial of capecitabine vs S-1 as first-line treatment in elderly patients with metastatic or recurrent unresectable gastric cancer. Br J Cancer. 2008;99(4):584-90. doi:10.1038/sj.bjc.6604536.

31. Kim GM, Jeung HC, Rha SY, Kim HS, Jung I, Nam BH, et al. A randomized phase II trial of S-1-oxaliplatin versus capecitabineoxaliplatin in advanced gastric cancer. Eur J Cancer. 2012;48(4): 518-26.

32. Koizumi W, Narahara H, Hara T, Takagane A, Akiya T, Takagi $\mathrm{M}$, et al. S-1 plus cisplatin versus S-1 alone for first-line treatment of advanced gastric cancer (SPIRITS trial): a phase III trial. Lancet Oncol. 2008;9(3):215-21. doi:10.1016/s1470-2045(08) 70035-4.

33. Narahara $\mathrm{H}$, Iishi $\mathrm{H}$, Imamura $\mathrm{H}$, Tsuburaya $\mathrm{A}$, Chin $\mathrm{K}$, Imamoto $\mathrm{H}$, et al. Randomized phase III study comparing the efficacy and safety of irinotecan plus S-1 with S-1 alone as first-line treatment for advanced gastric cancer (study GC0301/TOP-002). Gastric Cancer. 2011;14(1):72-80. doi:10.1007/s10120-011-0009-5.

34. Komatsu Y, Takahashi Y, Kimura Y, Oda H, Tajima Y, Tamura $\mathrm{S}$, et al. Randomized phase II trial of first-line treatment with tailored irinotecan and S-1 therapy versus S-1 monotherapy for advanced or recurrent gastric carcinoma (JFMC31-0301). Anticancer Drugs. 2011;22(6):576-83. doi:10.1097/CAD.0b013e328 $345 b 509$.

35. Wang X, Wang ML, Zhou LY, Lu XY, Yang JF, Yu HG. Randomized phase II study comparing paclitaxel with S-1 vs. S-1 as first-line treatment in patients with advanced gastric cancer. Clin Transl Oncol. 2013;15(10):836-42. doi:10.1007/s12094013-1012-6.

36. Chen WW, Wang F, Xu RH. Platinum-based versus non-platinum-based chemotherapy as first line treatment of inoperable, advanced gastric adenocarcinoma: a meta-analysis. PLoS One. 2013;8(7):e68974.

37. Dank M, Zaluski J, Barone C, Valvere V, Yalcin S, Peschel C, et al. Randomized phase III study comparing irinotecan combined with 5-fluorouracil and folinic acid to cisplatin combined with 5 -fluorouracil in chemotherapy naive patients with advanced adenocarcinoma of the stomach or esophagogastric junction. Ann Oncol. 2008;19(8):1450-7. doi:10.1093/annonc/mdn166.

38. Van Cutsem E, Moiseyenko VM, Tjulandin S, Majlis A, Constenla M, Boni C, et al. Phase III study of docetaxel and cisplatin plus fluorouracil compared with cisplatin and fluorouracil as firstline therapy for advanced gastric cancer: a report of the V25 study group. J Clin Oncol. 2006;24(31):4991-7. doi:10.1200/ JCO.2006.06.8429.

39. Chen XL, Chen XZ, Yang C, Liao YB, Li H, Wang L, et al. Docetaxel, cisplatin and fluorouracil (DCF) regimen compared with non-taxane-containing palliative chemotherapy for gastric carcinoma: a systematic review and meta-analysis. PLoS One. 2013;8(4). doi:10.1371/journal.pone.0060320.

40. Pozzo C, Barone C, Szanto J, Padi E, Peschel C, Bukki J, et al. Irinotecan in combination with 5-fluorouracil and folinic acid or with cisplatin in patients with advanced gastric or esophagealgastric junction adenocarcinoma: results of a randomized phase II study. Ann Oncol. 2004;15(12):1773-81. doi:10.1093/annonc/ $\operatorname{mdh} 473$.

41. Longley DB, Harkin DP, Johnston PG. 5-Fluorouracil: mechanisms of action and clinical strategies. Nat Rev Cancer. 2003;3:330-8. doi:10.1038/nrc1074. 